\title{
Doehlert Matrix-assisted optimization of Salmonella typhi Vi polysaccharide purification parameters
}

\author{
Garisala Mallikarjunara Rao ${ }^{1}$, Sathish Thadikamala ${ }^{2 *}$, Maram Chinna Eswaraiah ${ }^{3}$ \\ ${ }^{1}$ Department of Biotechnology, JNTU Hyderabad, Hyderabad, India. \\ ${ }^{2}$ Department of Pharmacology, Koringa College of Pharmacy, Kakinada, India. \\ ${ }^{3}$ Department of Pharmaceutical Biotechnology, Anurag Pharmacy Collage, Kodad, India.
}

\begin{tabular}{l}
\hline ARTICLE INFO \\
\hline Article history: \\
Received on: March 21, 2021 \\
Accepted on: May 19, 2021 \\
Available online: September 01, 2021 \\
\hline
\end{tabular}

\section{Key words: \\ Vi capsular polysaccharide, Salmonella typhi, typhoid vaccine, Doehlert design, response surface method, O-acetyl content}

\begin{abstract}
Typhoid fever is one of the prevalent pandemics across many developing countries caused by Salmonella typhi. Vi capsular polysaccharide obtained from S. typhi has been licensed for use as a vaccine for children aged 2 years and above to prevent typhoid fever. Production and purification of Vi polysaccharide play a vital role in the immunogenicity and cost of vaccines. Four critical parameters, cetrimide concentration ( $\% \mathrm{w} / \mathrm{v})$, sodium acetate concentration $(\% \mathrm{w} / \mathrm{v})$, alcohol concentration $(\% \mathrm{w} / \mathrm{v})$, and operational temperature $\left({ }^{\circ} \mathrm{C}\right)$, were optimized by response surface methodology (RSM). Doehlert design matrix, with 22 experimental runs, was used to optimize the selected parameters. All parameters were studied at different levels. With the help of RSM, by optimizing the selected variable conditions, onefold yield increased and the final purified Vi polysaccharide has noncompliance with the World Health Organization's standards.
\end{abstract}

\section{INTRODUCTION}

In the worldwide history of pandemic outbreaks, typhoid fever has a significant role. At present, many countries in Africa, Latin America, and South Asia are affected by the typhoid fever and the effect shows a serious impact on the society. According to the World Health Organization (WHO), recent data depict that every year worldwide 16-33 million typhoid fever cases are reported and among those 500,000-600,000 people are dying $[1,2]$. In humans, Salmonella enterica serovar typhi (Salmonella typhi) is a causative agent for typhoid fever $[3,4]$. The bacteria spreads through contaminated water and food [5,6]. It was reported that due to tourism also the bacteria spreads across the various European countries and USA [3]. The rate of risk of infection is severe in countries in the continents of Asia, Africa, and Latin America where poor sanitation is observed [7]. Typhoid fever is mostly observed in underaged children and school going children.

*Corresponding Author

Sathish Thadikamala, Department of Pharmacology, Koringa College of Pharmacy, Kakinada, India. E-mail: sathish.thadikamala@gmail.com
Fatal rate was observed among children in the age group of 1-2 years. A number of antibiotics have been used to treat typhoid fever. However, emerging multidrug-resistant strains of S. typhi make those antibiotic treatments unsuccessful $[5,6]$. The low costeffective vaccine is still the most effective way to control typhoid fever.

Currently, there are two quite distinct vaccines that are available in the market. One oral live attenuated vaccine that contain strain Ty21a and Vi polysaccharide vaccine administered parenterally [7]. The oral typhoid vaccine is given in the form of enteric capsules as three to four doses [8]. At present, the mechanism action of the oral vaccine is not fully understood [9]. Since it is administered through an oral route it activates the mucosal IgA and serum IgG and provides protection from typhoid bacteria [10,11]. The live attenuated bacteria lack the galE gene, are unable to synthesize the lipopolysaccharides (LPS) O-chain, and are unable to synthesis the capsular polysaccharide (CP) Vi antigen. Both LPS and CP are considered major targets for antibodies that lack these components and in the live attenuated vaccines they elicit less immunogenicity [12]. The efficacy of Ty21a enteric capsules after three doses is noted as $42 \%-67 \%$ at 3 years postvaccination $[13,14]$. 
CPs are composed of monosaccharides attached by glycosidic linkage. CPs have been proven to be excellent targets for bacterial vaccine development $[15,16]$. Vi capsular polysaccharide consist of polygalacturonic acid units linked linearly by $\alpha-1-4$ linkage, and it is $\mathrm{N}$-acetylated at $\mathrm{C} 2$ and $60 \%-90 \%$ O-acetylated at $\mathrm{C} 3$ of the galacturonic acid (Gal UA) residue [17,18]. The molecular weight and $\mathrm{O}$-acetylation content play vital roles in the immunogenicity and potency of Vi polysaccharide. After 28 days of injection of single dose of $25 \mu \mathrm{g}$ purified Vi polysaccharide given intramuscularly, it elicits $1 \mu \mathrm{g} / \mathrm{ml}$ anti typhoid IgG antibody in the serum $[17,18]$. The efficacy of Vi polysaccharide-based vaccines showed superior immunogenicity than live attenuated vaccines $[19,20]$.

The Vi polysaccharide is produced by the fermentation of S. typhi. Production and purification of Vi polysaccharide play an important role in the final price fixing of the vaccine. It is necessary to produce economically viable vaccines for low-income country people. It is important to avoid costly process purification methods such as chromatography and various capital equipment to reduce the final vaccine price. It is also important to produce the vaccine to meet the WHO's standards with less impurities and high content of Vi polysaccharide [20,21]. The precipitation method was found to be an economically viable method to purify the Vi polysaccharide [20]. Various researchers have developed various methods for purification of polysaccharide, among which the cetrimide method was found to be an effective and economically viable method. The current manuscript deals with the optimization of various precipitation parameters to enhance the yield. The statistical method of optimization was employed to enhance the Vi content. To the best of our knowledge, this is the first manuscript to publish the statistical method of optimization for the enhancement of $\mathrm{Vi}$ content and it is purity.

\section{MATERIALS AND METHODS}

\subsection{Bacterial Strain and Production of Vi Polysaccharide}

For the production of Vi polysaccharide, S. typhi Tystrain was employed. The culture was stored on solid Triple sugar iron (TSI) agar slants. It was revived from the TSI slants and grown on the shake flasks. From the flask, the culture grew on the 301 bioreactor (Sartorius). Soybean Casein Digest Medium $30 \mathrm{~g} / \mathrm{l}$ supplemented with $\mathrm{MgSO} 47 \mathrm{H}_{2} 02.5 \mathrm{~g} / \mathrm{ml}$, yeast extract $5 \mathrm{~g} / 1$, and trace elements (such as cobaltous chloride $0.043 \% \mathrm{w} / \mathrm{v}$, manganese chloride $0.26 \% \mathrm{w} / \mathrm{v}$, copper sulfate $0.038 \% \mathrm{w} / \mathrm{v}$, boric acid $0.053 \% \mathrm{w} / \mathrm{v}$, sodium molybdate $0.037 \% \mathrm{w} / \mathrm{v}$, and zinc acetate $0.26 \% \mathrm{w} / \mathrm{V}$ ) were used for cultivation of S. typhi. The media $\mathrm{pH}$ was maintained at 7.2 \pm 0.1 with $\mathrm{NaOH}$ and $\mathrm{HCl}$. The prepared media were sterilized by autoclaving at $121^{\circ} \mathrm{C}$ for 20 minutes. The sterile media inoculated with the $2 \%$ inoculum of $\mathrm{OD}_{590} 2$ and were incubated for 12 and 24 hours in the shaker and bioreactor, respectively.

\subsection{Clarification of Fermented Broth}

The fermentation broth was clarified by using the centrifuge, and the crude Vi polysaccharide was distributed into centrifuge bottles and centrifuged at $8000 \mathrm{rpm}$ for 30 minutes at $2^{\circ} \mathrm{C}-8^{\circ} \mathrm{C}$. The supernatant was collected in bottle and pellets were collected in a separate bottle. The supernatant was further concentrated up to 1.01 by using $30 \mathrm{kD}$ Tangential flow filtration (TFF) cassettes, followed by diafilteration against 10 volume change of purified water maintaining a constant retantate volume.

\subsection{Purification of Vi Polysaccharide}

Figure 1 shows the flow chart of the purification process. To the crude Vi polysaccharide, $10 \%$ of cetrimide was add slowly while stirring, so that the final concentration of cetrimide reached a desired concentration as per experimental conditions and was incubated in a refrigerator overnight. The centrifuged solution and pellets were collected and the supernatant was discarded. The obtained pellets were redissolved in $1 \mathrm{M} \mathrm{NaCl}$ solution and ethanol was added to reach a final concentration as per experimental conditions, and the mixture was incubated in a refrigerator overnight. Then, the centrifuged solution and pellets were collected and the supernatant was discarded.

The pellets were redissolved in $\mathrm{NaCl}$ solution and ethanol, and to this solution a desired amount of sodium acetate was added slowly while stirring after complete dissolution of sodium acetate to make up the final volume with ethanol or water to achieve desired concentration of ethanol in the solution. The precipitate was allowed to settle down at the bottom of the container, and without disturbing the precipitate the mixture was incubated in the refrigerator for 6 hours. At the end of incubation period, the crude Vi polysaccharide mixture was distributed into centrifuge bottles and centrifuge at $8000 \mathrm{rpm}$ for 30 minutes at $2^{\circ} \mathrm{C}-8^{\circ} \mathrm{C}$. The supernatant was collected and the pellets were discarded.

To the collected supernatant solution a desired amount of ethanol was slowly added while stirring. The precipitate was allowed to settle down at the bottom of the container. The precipitate mixture was incubated in the refrigerator overnight. At the end of the incubation period, the Vi polysaccharide mixture was distributed into centrifuge bottles and centrifuged at $8000 \mathrm{rpm}$ for 30 minutes at $2^{\circ} \mathrm{C}-8^{\circ} \mathrm{C}$. The pellets contained the desired purified $\mathrm{Vi}$ polysaccharide. The pellets were redissolved $1 \mathrm{M} \mathrm{NaCl}$ solution and additional concentrate and the $\mathrm{NaCl}$ was removed by diafiltration.

\subsection{Diafiltration and Sterile Filtration of Vi Polysaccharide}

The purified Vi polysaccharide was further concentrated by using $100 \mathrm{kD}$ TFF cassettes, followed by diafiltration against 10 volume change of purified water maintaining a constant retantate volume to ensure maximal recovery of Vi polysaccharide. Finally, the sterile solution was filtered through a $0.2 \mu$ filter and stored in a sterile container.

\subsection{Optimization of Purification Conditions by Response Surface Methodology (RSM)}

RSM using Doehlert design was applied for optimization of purification conditions. Four parameters, cetrimide concentration $(\% \mathrm{w} / \mathrm{v})(\mathrm{C})$, sodium acetate concentration (\% w/v) (S), final alcohol concentration $(\% \mathrm{v} / \mathrm{v})(\mathrm{A})$, and process temperature $\left({ }^{\circ} \mathrm{C}\right)$ $(\mathrm{T})$, were selected as independent variables and $\mathrm{O}$-acetyl content (mmol/g of $\mathrm{Vi}$ ) was a dependent response variable. Doehlert's design for four variables was chosen from Ferreira et al.'s [22] 


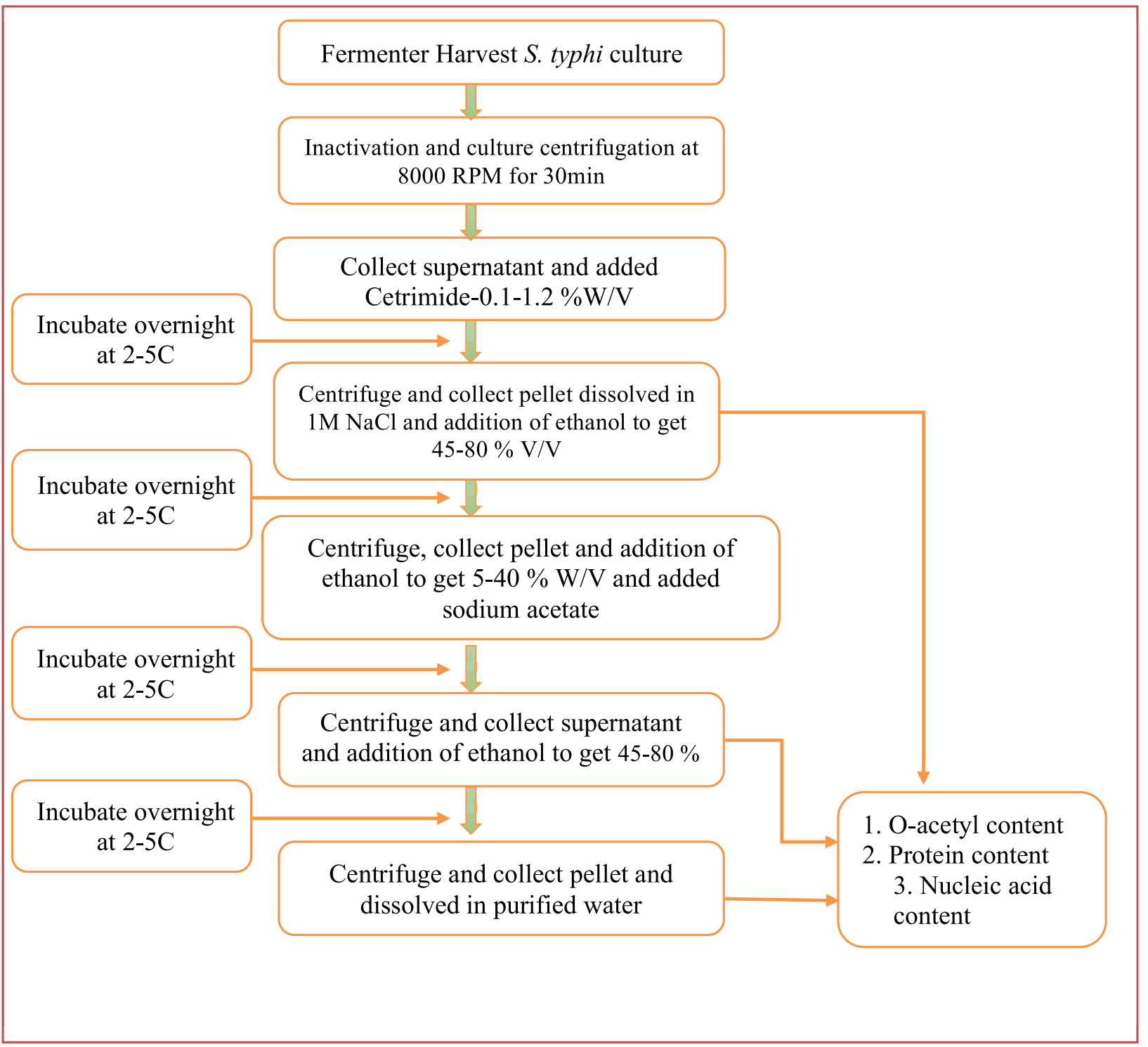

Figure 1: Flow chart of the Vi polysaccharide purification process.

study and added one additional central point. The experimental conditions were selected according to the design. Cetrimide concentration was chosen at five levels, sodium acetate and ethanol selected at seven levels, and temperature was selected at three levels with a total of 22 experimental runs. The experimental plan of RSM in both coded and real values of all independent variables is presented in Table 1 .

The obtained Vi polysaccharide, in terms of O-acetyl content with respect to purification conditions, was analyzed, and the response surface model given by Equation (1) was fitted with multiple regressions through the least squares method as follows:

$Y_{i}=\beta_{0}+\sum_{i=1}^{k} \beta_{i} x_{i}+\sum_{i=1}^{k} \beta_{i i} x_{i}{ }^{2}+\sum_{i}^{i} \sum_{j}^{k} \beta_{i j} x_{i} x_{i}----(1)$

where $Y_{i}$ is the predicted $\mathrm{O}$-acetyl content $\left(Y_{i}\right)$ taken as a response, $x_{i-} x_{j}$ are input variables which influence the response variable $Y, \beta_{0}$ is the offset term, $\beta_{i}$ is the $i$ th linear coefficient, $\beta_{i i}$ the $i$ th quadratic coefficient, and $\beta_{i j}$ is the interaction coefficients. The significance of the selected parameters is evaluated based on $p$-values and $F$-test with unequal variance $(p<0.05, p<0.01$, and $p<0.001)$. Trail version of Design-Expert-12 (State Ease Company, Minneapolis, $\mathrm{MN}$ ) was used in this study.

\subsection{Analytical Methods}

Vi polysaccharide was estimated according to Hestrin [23]. The protein content was estimated by Lowry et al. [24]. UV spectroscopy method was used for estimation of nucleic content [20].

\section{RESULTS AND DISCUSSION}

For effective purification of the Vi polysaccharide, it is essential to optimize all the purification conditions. Initially, critical purification parameters were identified and optimized by the conventional method 
(data not shown). From these studies, it was observed that cetrimide, sodium acetate concentrations, final alcohol concentration, and temperature play a vital role in the Vi polysaccharide purification. The current focus on polysaccharide purification research is oriented toward the optimization of selected parameters by statistical methods. Various statistical methods, such as the Plackett-Burman design [25], RSM with the Box-Benhan design, central composite design [26], Doehlert's design [27], and mixture designs [28], are employed in the optimization of various culture conditions. However, this is the first research employing the RSM with Doehlert's design to optimize the Vi polysaccharide purification variables.

Table 1 shows the purification of $\mathrm{Vi}$ polysaccharide ranged between 1.23 and $3.01 \mathrm{O}$-acetyl content ( $\mathrm{mmol} / \mathrm{g}$ of Vi), indicating the significance of selected factors and their concentrations on the purity of polysaccharide. Multiple regression analysis carried out by taking $\mathrm{O}$-acetyl content as the response and selected parameters as independent variables. The regression coefficient $\left(R^{2}\right)$ value was considered for the accuracy of the analysis, and it was preferred that $R^{2}$ value nearer to one is desirable. In the current experiment, the $R^{2}$ value obtained is 0.9963 , indicating that $99.63 \%$ of the variability in the purification process can be explained by the model.
Furthermore, the observed adjusted $R^{2}$ value of 0.9889 signifies the model [26]. The coefficient of variance (CV) is a measure of the precision and accuracy of experiments. A lower $\mathrm{CV}$ value indicates better accuracy [26]. In the current experiment, the CV was $2.71 \%$, which indicates good precision and reliability of the experiments conducted. The signal-to-noise ratio can be measured by adequate precision value. A value above 4 is desirable, and in the current experiment the adequate precision value of 38.377 indicated the current model can be used for further optimization.

Figure 2 shows the correlation between the experimental values and predicted values. In Figure 1, all data points near to the fitted line indicate the best correlation between the observed and predicted values [27].

Based on multiple regression analysis data, a second-order polynomial regression equation was constructed, which relates the studied parameters and purity of Vi polysaccharide empirically.

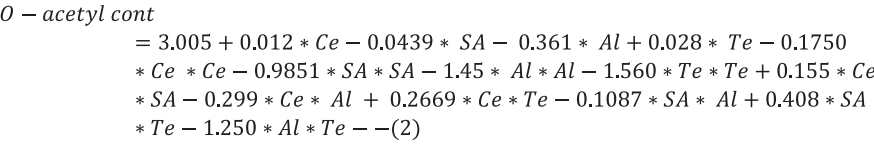

Table 1: Doehlert design for optimization of Vi polysaccharide along with experimental results and predicted values.

\begin{tabular}{|c|c|c|c|c|c|c|c|}
\hline \multirow{2}{*}{$\begin{array}{l}\text { S. } \\
\text { No }\end{array}$} & \multirow{2}{*}{$\begin{array}{c}\text { Cetrimide concentration } \\
(\% W / V)(\mathrm{Ce})\end{array}$} & \multirow{2}{*}{$\begin{array}{c}\text { Sodium acetate } \\
\text { conc }(\% W / V)(\mathrm{SA})\end{array}$} & \multirow{2}{*}{$\begin{array}{l}\text { Alcohol conc } \\
(\% V / V)(\mathrm{AL})\end{array}$} & \multirow{2}{*}{$\begin{array}{c}\text { Temperature }\left({ }^{\circ} \mathrm{C}\right) \\
(\mathrm{Te})\end{array}$} & \multicolumn{3}{|c|}{ O-acetyl content (mmol/g of $\mathrm{Vi})$} \\
\hline & & & & & Experimental & Predicted & Error \\
\hline 1 & $0(0.6)$ & $0(20)$ & $0(60)$ & $0(5)$ & 3.00 & 3.01 & -0.005 \\
\hline 2 & $1(1.2)$ & $0(20)$ & $0(60)$ & $0(5)$ & 2.87 & 2.84 & 0.028 \\
\hline 3 & $0.5(0.9)$ & $0.866(40)$ & $0(60)$ & $0(5)$ & 2.21 & 2.26 & -0.048 \\
\hline 4 & $0.5(0.9)$ & $0.289(25)$ & $0.817(65)$ & $0(5)$ & 1.45 & 1.48 & -0.033 \\
\hline 5 & $0.5(0.9)$ & $0.289(25)$ & $0.204(80)$ & $0.791(15)$ & 1.82 & 1.77 & 0.053 \\
\hline 6 & $-1(0.1)$ & $0(20)$ & $0(60)$ & $0(5)$ & 2.79 & 2.82 & -0.028 \\
\hline 7 & $-0.5(0.3)$ & $-0.866(5)$ & $0(60)$ & $0(5)$ & 2.37 & 2.32 & 0.048 \\
\hline 8 & $-0.5(0.3)$ & $0.289(15)$ & $-0.817(50)$ & $0(5)$ & 2.12 & 2.09 & 0.033 \\
\hline 9 & $-0.5(0.3)$ & $0.289(15)$ & $-0.204(45)$ & $-0.791(2)$ & 1.83 & 1.88 & -0.053 \\
\hline 10 & $0.5(0.9)$ & $-0.866(5)$ & $0(60)$ & $0(5)$ & 2.18 & 2.20 & -0.019 \\
\hline 11 & $0.5(0.9)$ & $0.289(15)$ & $-0.817(50)$ & $0(5)$ & 2.30 & 2.30 & 0.001 \\
\hline 12 & $0.5(0.9)$ & $0.289(15)$ & $-0.204(45)$ & $-0.791(2)$ & 1.69 & 1.70 & -0.01 \\
\hline 13 & $-0.5(0.3)$ & $0.866(40)$ & $0(60)$ & $0(5)$ & 2.13 & 2.11 & 0.019 \\
\hline 14 & $0(0.6)$ & $0.577(30)$ & $-0.817(50)$ & $0(5)$ & 2.05 & 2.03 & 0.02 \\
\hline 15 & $0(0.6)$ & $0.577(30)$ & $-0.204(45)$ & $-0.791(2)$ & 1.30 & 1.29 & 0.009 \\
\hline 16 & $-0.5(0.3)$ & $0.289(25)$ & $0.817(65)$ & $0(5)$ & 1.67 & 1.67 & -0.001 \\
\hline 17 & $0(0.6)$ & $-0.577(10)$ & $0.817(65)$ & $0(5)$ & 1.47 & 1.49 & -0.02 \\
\hline 18 & $0(0.6)$ & $0(20)$ & $0.613(70)$ & $-0.791(2)$ & 1.90 & 1.85 & 0.054 \\
\hline 19 & $-0.5(0.3)$ & $0.289(25)$ & $0.204(80)$ & $0.791(15)$ & 1.57 & 1.56 & 0.01 \\
\hline 20 & $0(0.6)$ & $-0.577(10)$ & $0.204(80)$ & $0.791(15)$ & 1.23 & 1.24 & -0.009 \\
\hline 21 & $0(0.6)$ & $0(20)$ & $-0.613(50)$ & $0.791(15)$ & 2.28 & 2.33 & -0.054 \\
\hline 22 & $0(0.6)$ & $0(20)$ & $0(60)$ & $0(5)$ & 3.01 & 3.01 & 0.005 \\
\hline
\end{tabular}

Values in parenthesis are real values. The error in the table is the difference between the experimental results and predicted O-acetyl content (mmol/gm of Vi) 
Based on the $p, F$, and $t$-values, the coefficients were selected. Table 2 shows the coefficient values and their corresponding $p$, $F$, and $t$-values. The coefficients having a $p$-value below 0.05 and a high $F$-value are considered significant terms. Based on these criteria, linear terms of cetrimide, alcohol content, temperature, and interaction terms of sodium acetate with cetrimide and alcohol are insignificant. The square terms of temperature has the highest effect among all selected parameters, which indicates that temperature has a critical role in the Vi purification process. The square terms of cetrimide, alcohol content, and sodium acetate

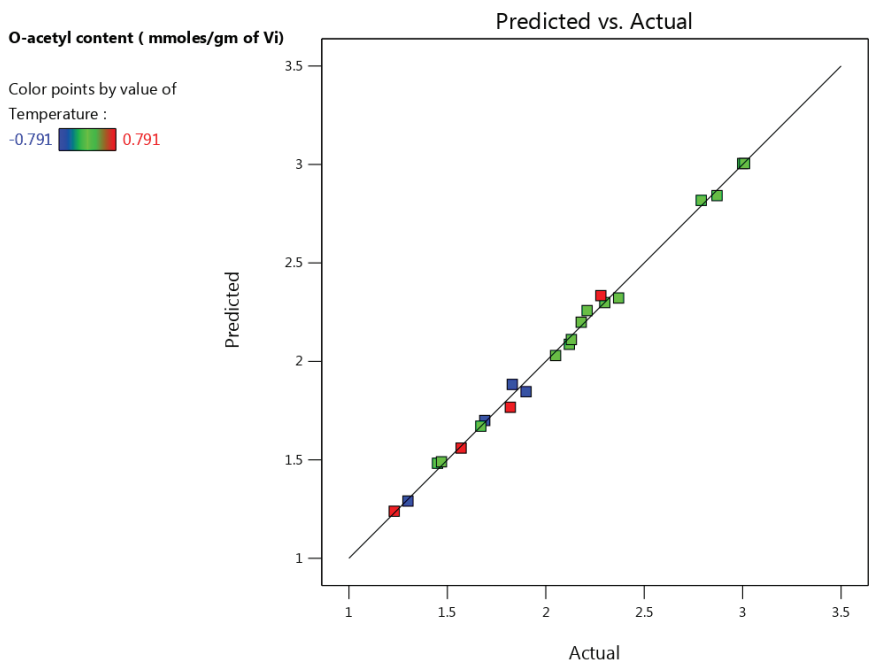

Figure 2: Correlation between experimental results (actual) and modelpredicted $\mathrm{O}$-acetyl content values. concentration have more effect value. The parameters which have more effect value in square terms than linear terms are critical to the process, and a small variation in those parameters values has a significant effect on the final process. In this study, all selected parameters have more effect at square terms than linear terms, which indicates that all selected parameters and their levels are important for the purity of Vi polysaccharide.

\subsection{Evaluation of Response Surface}

The surface (3D) plots along with contour plots were (2D) generated by using the regression equation developed (Eq. 3). The $3 \mathrm{D}$ plots with $2 \mathrm{D}$ contour plots visualized the interaction influence of the selected two parameters on Vi polysaccharide purification. In these plots, two parameters were changed at different levels, whereas other parameters were kept constant at central value. The highest values of polysaccharide could be assessed with combinations of the two variables that were close to the central points. Figure 3 shows the surface plots with images of contour plots of selected variables and their influence on Vi polysaccharide recovery.

Figure $3 \mathrm{~A}-\mathrm{C}$ shows the cetrimide interaction with other selected three parameters for the purification of $\mathrm{O}$-acety content. Figure $3 \mathrm{~A}-\mathrm{C}$ shows that the contours are slightly inclined toward the $y$-axis, indicating that the cetrimide concentration was slightly influenced by sodium acetate, alcohol concentration, and temperature. Figure 3D and E shows circular contours indicating that sodium acetate concentration was independent of alcohol concentration and temperature. Interaction of alcohol content with temperature depicts that alcohol content depended on process temperature (Fig. 3F).

Table 2: Coefficients and analysis of variance.

\begin{tabular}{ccccccc} 
& Coefficients & Sum of squares & df & Mean square & $\boldsymbol{F}$-value & $\boldsymbol{p}$-value \\
Model & & 5.85 & 14 & 0.418 & 134.6 & $<0.0001$ \\
Constant & 3.01 & - & - & - & - & $<0.0001$ \\
Ce & 3.005 & 0.0007 & 1 & 0.0007 & 0.2318 & 0.6449 \\
SA & 0.012 & 0.0096 & 1 & 0.0096 & 3.1 & 0.1217 \\
AL & -0.04388 & 0.6541 & 1 & 0.6541 & 210.61 & $<0.0001$ \\
Te & -0.36147 & 0.004 & 1 & 0.004 & 1.29 & 0.2926 \\
Ce*Ce & -1.25057 & 0.0306 & 1 & 0.0306 & 9.86 & 0.0164 \\
SA*SA & -0.175 & 0.9702 & 1 & 0.9702 & 312.4 & $<0.0001$ \\
AL*AL & -0.98506 & 2.3 & 1 & 2.3 & 741.02 & $<0.0001$ \\
Te*Te & -1.45067 & 2.87 & 1 & 2.87 & 924.26 & $<0.0001$ \\
Ce*SA & 0.028345 & 0.0182 & 1 & 0.0182 & 5.87 & 0.0459 \\
Ce*AL & 0.155889 & 0.054 & 1 & 0.054 & 17.4 & 0.0042 \\
Ce*Te & -0.29994 & 0.0396 & 1 & 0.0396 & 12.76 & 0.0091 \\
SA*AL & 0.266923 & 0.0071 & 1 & 0.0071 & 2.29 & 0.1742 \\
SA*Te & -0.10873 & 0.0928 & 1 & 0.0928 & 29.88 & 0.0009 \\
AL*Te & 0.408431 & 0.8707 & 1 & 0.8707 & 280.36 & $<0.0001$ \\
Residual & -1.56032 & 0.0217 & 7 & 0.0031 & 0.0036 & 72.3 \\
Lack of fit & & 0.0217 & 6 & 1 & 0 & 0.0898 \\
Pure error & & 0 & 21 & & & \\
Total & & 5.87 & & & \\
\hline
\end{tabular}



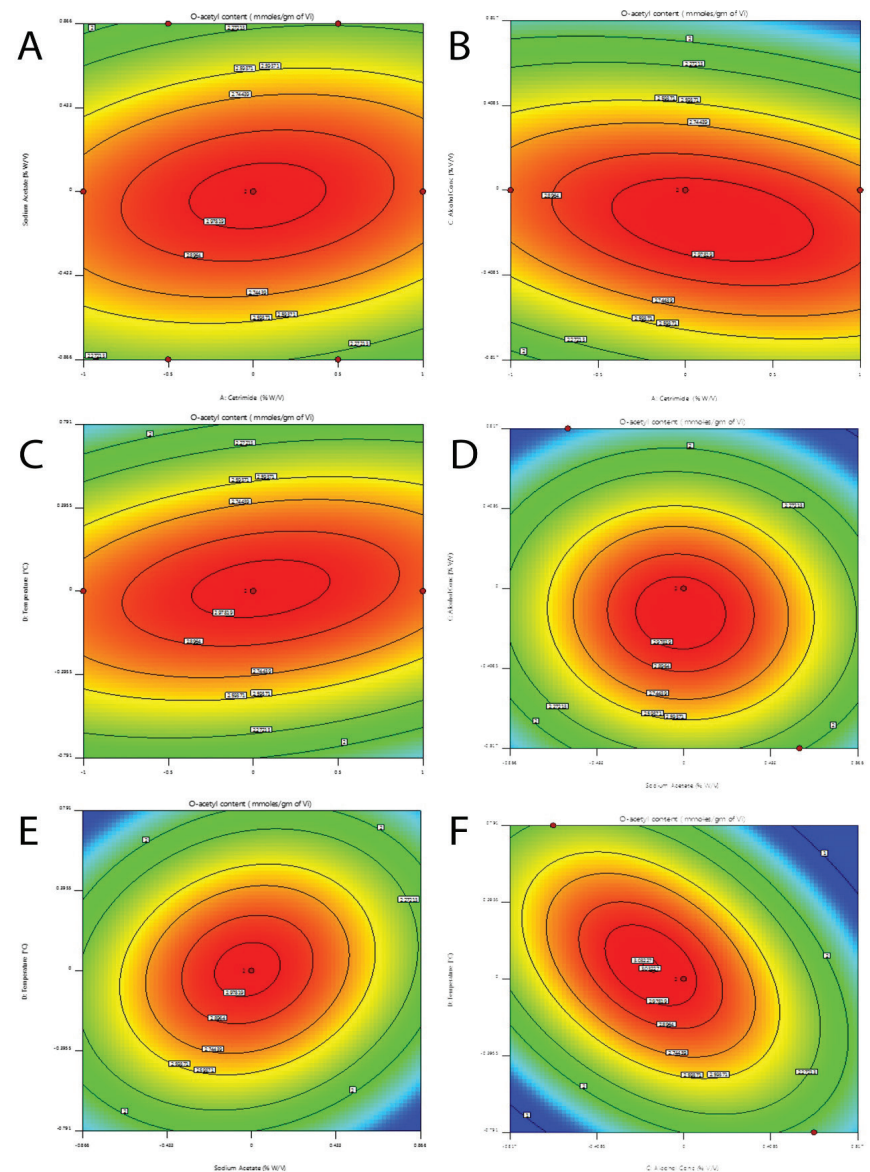

Figure 3: 3D surface plots with contours of selected parameters and their interaction influence on $\mathrm{O}$-acetyal content recovery. (A) Cetrimide versus sodium acetate, (B) cetrimide versus alcohol content, $(C)$ cetrimide versus temperature, (D) sodium acetate versus alcohol content, (E) sodium acetate versus temperature, and $(\mathrm{F})$ alcohol content versus temperature.

\subsection{Validation of Model}

To obtain the optimum conditions, Equation (2) was used. Solving Equation (2) by numerical methods, it has given the optimum condition, i.e., cetrimide concentration $0.7 \% \mathrm{w} / \mathrm{v}$, sodium acetate concentration $28 \% \mathrm{w} / \mathrm{v}$, final alcohol concentration at $55 \% \mathrm{v} / \mathrm{v}$, and temperature at $5.3^{\circ} \mathrm{C}$. At these conditions, the predicted O-acety content was 3.1 ( $\mathrm{mmol} / \mathrm{g}$ of $\mathrm{Vi})$; however by conducting the experiments at the predicted conditions $3.15 \mathrm{mmol} / \mathrm{g}$ of $\mathrm{Vi}$ O-acetyl content was observed, at these conditions $3.066 \mathrm{mg} / \mathrm{g}$ of $\mathrm{Vi}$ of protein and $4.135 \mathrm{mg} / \mathrm{g}$ of $\mathrm{Vi}$ nucleic acid content was observed. The amount of protein and nucleic acid impurities are below the WHO specifications. Overall, the study indicates that about onefold increase in Vi polysaccharide was observed compared with the conventional optimization methods.

\section{CONCLUSION}

The current study describes that precipitation is the best method for purification of polysaccharides. Finding the critical parameters is important for process development. In this study, four critical parameters, namely cetrimide concentration, sodium acetate concentration, alcohol concentration, and temperature, were identified for Vi polysaccharide purification. Statistical methods of optimization are effective with less number of experiments. Doehlert matrix method is diverse than the regular Box-Behnkhen and central composite design methods in RSM. This method has freedom of variables at individual levels. The total number of experiments is less with high variability in specific parameters. Overall, onefold improvement of Vi polysaccharide recovery with high purity as per the WHO's specifications was achieved.

\section{ACKNOWLEDGMENTS}

The author Mallikarjunara Rao is thankful to the Head of Department of Biotechnology, JNTU Hyderabad, Kukatpally, Hyderabad, Telangana, for allowing to use the departmental facilities which were acquired previously out of the grants from various projects.

\section{AUTHORS' CONTRIBUTIONS}

All authors made substantial contributions to the conception and design, acquisition of data, and analysis and interpretation of data; they took part in drafting the article and revising it critically for important intellectual content; they agreed to submit the current journal; they gave their final approval of the version to be published and agree to be accountable for all aspects of the work. All the authors are eligible to be an author as per the International Committee of Medical Journal Editors' requirements/guidelines.

\section{ETHICAL APPROVAL}

This study does not involve experiments on animals or human subjects.

\section{CONFLICT OF INTEREST}

The authors report no financial or any other conflicts of interest in this work.

\section{FUNDING}

There is no funding to report.

\section{REFERENCES}

1. Haque S, Sengupta S, Gupta D, Bhan MK, Kumar R, Khan A, et al. S. typhi derived OmpC peptide conjugated with Vi-polysaccharide evokes better immune response than free Vi-polysaccharide in mice. Biologicals 2019; 62:50-6.

2. Initiative for Vaccine Research. Typhoid fever at Available via http:// www.who.int/ vaccineresearch/diseases/diarrhoeal/en/index7.html (Accessed 23 August 2007).

3. Ackers ML, Puhr ND, Tauxe RV, Mintz ED. Laboratory-based surveillance of Salmonella serotype typhi infections in the United States: antimicrobial resistance on the rise. J Am Med Assoc 2000; 20:2668-73.

4. Lawrence J, Jones J. Final report: pilot of enhanced enteric fever surveillance in England. Health Protection Agency, London, UK, 2008.

5. Sinha A, Sazawal S, Kumar R, Sood S, Reddaiah VP, Singh B. Typhoidfever in children aged less than 5 years. Lancet 1999; 354(9180):734-7. 


$$
\text { 2021;9(05):141-147 }
$$

6. Brooks WA, Hossain A, Goswami D, Sharmeen AT, Nahar K, Alam $\mathrm{K}$. Bacteremictyphoid fever in children in an urban slum, Bangladesh. Emerg Infect Dis 2005; 11:326-9.

7. Rigsby P, Beamish E, Hockley J, Atkinson E, Hitri K, Jones E, et al. Evaluation of a standardised Vi poly-l-lysine ELISA for serology of Vi capsular polysaccharide antibodies. Biologicals 2020; 66:21-9.

8. Staats HF, Kirwan SM, Whisnant CC, Stephenson JL, Wagener DK, Majumder PP. Development of a bead immunoassay to measure $\mathrm{Vi}$ polysaccharide-specific serum IgG after vaccination with the Salmonella enterica Serovar typhi Vi polysaccharide. Clin Vaccine Immunol 2010; 17:412-19.

9. Kalra S, Midha K, Shekhawat BPS. Production and purification of Vi polysaccharide from Salmonella enterica typhimurium (MTCC98). Biochem Indian J 2016; 10:43-51.

10. Germanier R, Furer E. Isolation and characterization of Gal-e mutanat ty 21 a of salmonella-typhi candidate strain for a live, oral Typhoid Vaccine. J Infect Dis 1975; 131:553-8.

11. Raffatellu M, Wilson RP, Winter SE, Baumler AJ. Clinical pathogenesis of typhoid fever. J Infect Dev Ctries 2008; 2:260-66.

12. Islam MT, Im J, Ahmmed F, Kim DR, Khan AI, Zaman K, et al. Use of typhoid Vi-polysaccharide vaccine as a vaccine probe to delineate clinical criteria for typhoid fever. Am J Trop Med Hyg 2020; 103: 665-71.

13. Engels EA, Lau J. Vaccines for preventing typhoid fever. Cochrane Database Syst Rev 2000; 2:CD001261.

14. Whitaker JA, Franco-Paredes C, Rio C, Edupuganti S. Rethinking typhoid fever vaccines: implications for travelers and people living in highly endemics areas. J Travel Med 2009; 16:46-52.

15. Szu SC, Li XR, Stone AL, Robbins JB. Relation between structure and immunologic properties of the Vi capsular polysaccharide. Infect Immun 1991; 59:4555-61.

16. Rijpkema S, Durrani Z, Lemercinier X, Jones C. Detection of O-acetylated Vi polysaccharide of Salmonella enterica subspecies typhi by enzyme immunoassay. Biologicals 2004; 32:11-6.

17. Jarvis FG, Mesenko MT, Martin DG, Perrine TD. Physicochemical properties of the Vi antigen before and after mild alkaline hydrolysis. J Bacteriol 1967; 94:1406-10.

18. Szewczyk B, Taylor A. Immunochemical properties of Vi antigen from Salmonella typhi Ty2: presence of two antigenic determinants. Infect Immun 1980; 29:539-44.

19. Landy M, Gaines S, Seal JR, Whiteside SE. Antibody responses of man to three types of antityphoid immunizing agents: heat-phenol fluid vaccine, acetonedehydrated vaccine, and isolated $\mathrm{Vi}$ and $\mathrm{O}$ antigens. Am J Public Health Nations Health 1954; 44:1572-79.

20. Kothari S, Kothari N, Kim JA, Lee E, Yoon YK, An SJ et al., A novel method for purification of Vi capsular polysaccharide produced by Salmonella enterica subspecies enterica serovar Typhi. Vaccine 2013; 31:4714-19.

21. WHO. WHO technical reports series, No. 840, Requirements for Vi polysaccharide typhoid vaccine. WHO, Geneva, Switzerland, 1994.

22. Ferreira SLC, dos Santos WNL, Neto BB, Quintella CM, BosqueSendra JM. Doehlert matrix: a chemometric tool for analytical chemistry-review. Talanta 2004; 63:1061-7.

23. Hestrin S. The reaction of acetylcholine and other carboxylic acid derivatives with hydroxylamine, and its analytical application. J Biol Chem 1949; 180:249-61.

24. Lowry OH, Rosebrough NJ, Farr AL. Randall RJ. Protein measurement with Folin phenol reagent. J Biol Chem 1951; 193:265-75.

25. Hymavathi M, Sathish T, Rao CS, Prakasham RS. Enhancement of L-asparaginase production by isolated Bacillus circulans (MTCC 8574) using response surface methodology. Appl Biochem Biotechnol 2009; 159:191-98.

26. Hymavathi M, Sathish T, Brahmaiah P, Prakasham RS. Impact of carbon and nitrogen sources on L-asparaginase production by isolated Bacillus circulan (MTCC8574): application of Plackett-Burman design. Chem Biochem Eng Q 2010; 24:473-80.

27. Sathish T, Kumar KP, Teja BB, Bobbarala V, Prakasham RS Enhancement of protein hydrolysates production by using Doehlertdesign approach. J Pharm Res 2009; 2:1889-92.

28. Sathish T, Lakshmi GS, Rao CS, Brahmaiah P, Prakasham RS. Mixture design as first step for improved glutaminase production in solid-state fermentation by isolated Bacillus sp. RSP-GLU. Lett Appl Microbiol 2008; 47:256-62.

How to cite this article:

Rao GM, Thadikamala S, Eswaraiah MC. Doehlert matrixassisted optimization of Salmonella typhi Vi polysaccharide purification parameters. J Appl Biol Biotech 2021; 9(05): 141-147. 